\title{
On the Design of MIMO Block-Fading Channels With Feedback-Link Capacity Constraint
}

\author{
Vincent Lau, Senior Member, IEEE, Youjian Liu, Member, IEEE, and Tai-Ann Chen, Member, IEEE
}

\begin{abstract}
In this paper, we propose a combined adaptive power control and beamforming framework for optimizing multiple-input/multiple-output (MIMO) link capacity in the presence of feedback-link capacity constraint. The feedback ${ }^{1}$ is assumed to be noiseless and causal with a feedback capacity constraint in terms of maximum number of feedback bits per fading block. We show that the hybrid design could achieve the optimal MIMO link capacity, and we derive a computationally efficient algorithm to search for the optimal design under a specific average power constraint. Finally, we shall illustrate that a minimum mean-square error spatial processor with a successive interference canceller at the receiver could be used to realize the optimal capacity. We found that feedback effectively enhances the forward channel capacity for all signal-to-noise ratio (SNR) values when the number of transmit antennas $\left(n_{T}\right)$ is larger than the number of receive antennas $\left(n_{R}\right)$. The SNR gain with feedback is contributed by focusing transmission power on active eigenchannel and temporal power waterfilling. The former factor contributed, at most, $10 \log _{10}\left(n_{T} / n_{R}\right)$ dB SNR gain when $n_{T}>n_{R}$, while the latter factor's SNR gain is significant only for low SNR values.
\end{abstract}

Index Terms-Communication theory with partial feedback, multiple-input/multiple-output (MIMO) link with partial feedback.

\section{INTRODUCTION}

$\mathbf{W}$ HILE SIDE information at the receiver is shown to enhance the single-input/single-output (SISO) link capacity [1] in fading channels, side information at the transmitter is also very useful. For example, [2] investigated the benefit of perfect side information at the transmitter side. Complete and causal feedback was assumed, and the results illustrated that the feedback capacity could be expressed as that of a memoryless channel without side information at both the transmitter [channel state information at transmitter (CSIT)] and the receiver [channel state information at receiver (CSIR)]. In [3]-[5], the benefits of having perfect side information at both the transmitter and the receiver of a SISO link is demonstrated. The performance degradation as a result of imperfect feedback, such as feedback delay, feedback noise, and feedback quantization have been studied in [6] and [7]. From

Paper approved by R. Raheli, the Editor for Detection, Equalization, and Coding of the IEEE Communications Society. Manuscript received November 28, 2002; revised April 9, 2003 and July 11, 2003.

V. Lau is with the Department of Electrical and Electronic Engineering, University of Hong Kong, Hong Kong (e-mail: knlau@ieee.org).

Y. Liu is with the Department of Electrical and Computer Engineering, University of Colorado, Boulder, CO 80309 USA (e-mail: eugeneliu@ieee.org).

T.-A. Chen is with Bell Labs, Lucent Technologies, Whippany, NJ 07981 USA (e-mail: tac@ieee.org).

Digital Object Identifier 10.1109/TCOMM.2003.822171

${ }^{1}$ The feedback channel is used to carry channel state information only. all the previous investigations, we know that the gain in side information feedback to the SISO transmitter is contributed by power adaptation, so that temporal power waterfilling could be employed at the transmitter with respect to an average transmit power constraint. Yet, for block-fading channels, where the encoding and decoding frames span over many fading blocks, the capacity gain is shown to be small, especially for large signal-to-noise ratios (SNRs).

On the other hand, the usage of CSIT at the multiple-input/multiple-output (MIMO) transmitter is more than power adaptation (temporal power waterfilling). For instance, the optimal MIMO transmission scheme with no CSIT is shown to be a bank of $n_{T}$ channel encoders [8]. On the other hand, the optimal MIMO transmission scheme with perfect CSIT is shown to be a cascade of channel encoder bank, adaptive power control matrix, and an eigen-beamforming matrix. These are illustrated in Fig. 1. The adaptive power-control matrix is to perform temporal and spatial waterfilling on an individual eigenchannel. ${ }^{2}$ The eigen-beamforming matrix, on the other hand, is to decompose the original $n_{R} \times n_{T}$ channel matrix $^{3}$ into $n=\min \left[n_{R}, n_{T}\right]$ decoupled eigenchannels, so that information carried along each eigenchannel will not interfere with each other at the receiver. However, all the above schemes require full feedback of the channel matrix, which induces a large feedback-capacity requirement, especially when $n_{T}$ or $n_{R}$ is large. Hence, this motivates the research on the partial feedback performance for MIMO systems.

In the past literature, there are several common approaches used to investigate the MIMO partial feedback problems. One common approach is to impose some artificial constraint on the transmission and feedback strategy and perform optimization based on the imposed constraint. For example, multipleinput/single-output (MISO) transmission with specific forms of partial power feedback is investigated in [9]. In [10], the optimal MIMO multiuser design with partial power feedback constraint is investigated. Performance of MIMO schemes with other specific forms of partial feedback are investigated in [11] and [12]. On the other hand, another common approach used in the literature is to design the system assuming the availability of full feedback, and study the performance degradation in the presence of imperfect feedback (such as feedback-link capacity constraint) [13]. All the approaches do not address the general design problem of the optimal feedback strategy and the optimal transmission strategy in the presence of a feedback-link capacity constraint. In this paper, we propose a hybrid beamforming and

\footnotetext{
2"Eigenchannel" refers to the independent spatial channels intrinsic in the multiple-antenna link.

${ }^{3} n_{T}$ is the number of transmit antennas. $n_{R}$ is the number of receive antennas.
} 


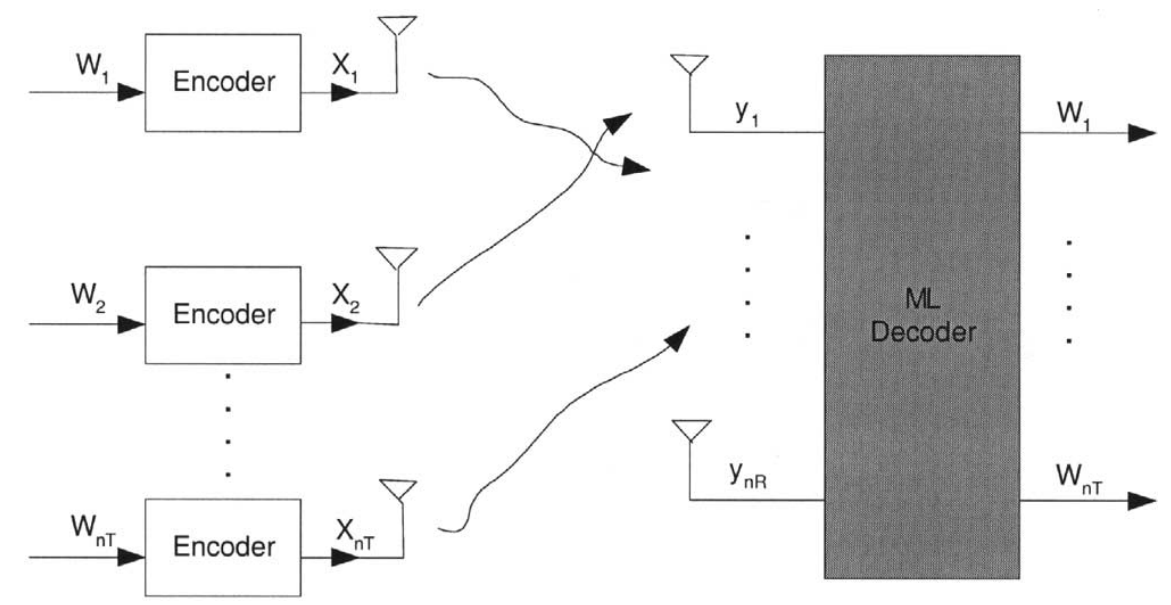

(a)

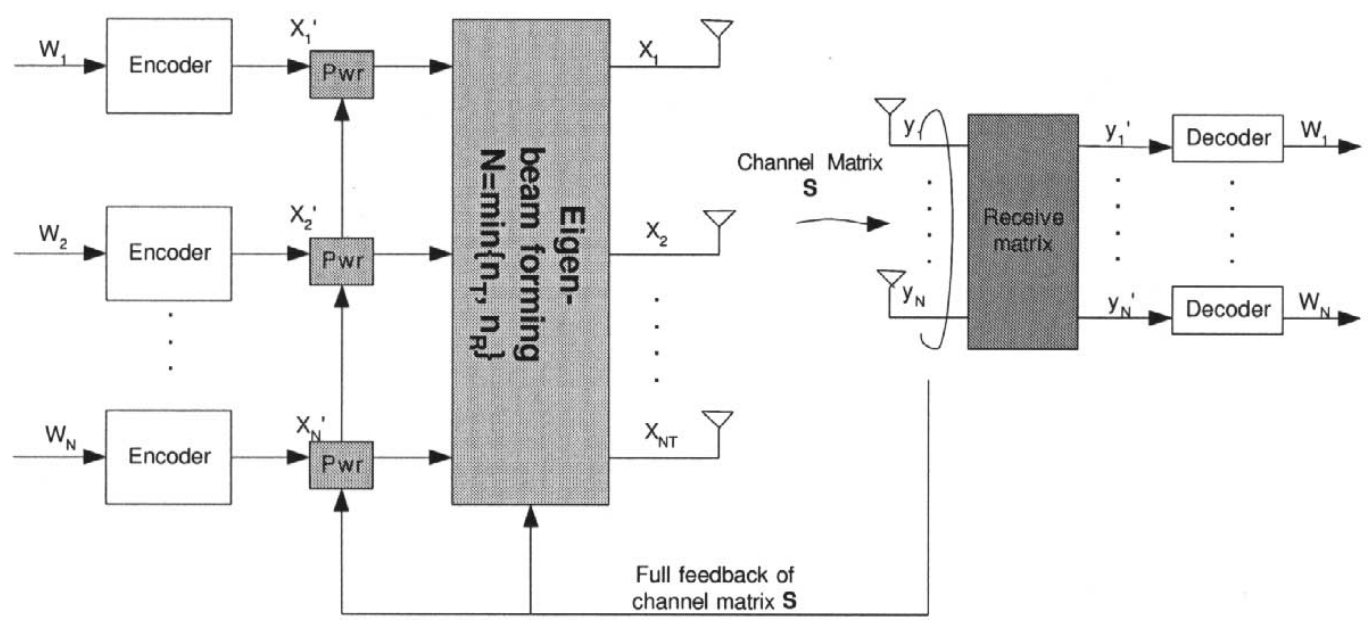

(b)

Fig. 1. Optimal architecture of MIMO transmission strategy for no CSIT and perfect CSIT.

adaptive power-control design framework, and we show that this architecture could achieve the optimal link capacity under feedback-link capacity constraint. Furthermore, a computationally efficient offline algorithm (Lloyd's algorithm) is proposed to find out the optimal parameters of the framework with respect to specific average power constraint. We also show that a receiver architecture employing minimum mean-square error (MMSE) spatial processing with successive interference cancellation (SIC) could be used to achieve the optimal capacity as well.

We consider a MIMO block-fading channel model where the channel state remains quasi-static within a fading block, but becomes independent across a different fading block. There are $n_{T}$ transmit antennas and $n_{R}$ receive antennas. Encoding and decoding are done across multiple fading blocks, as illustrated in Fig. 2, and hence, in the limit of large block length, nonzero ergodic capacity could be achieved. The channel matrix is estimated at the receiver and fed back to the transmitter (CSIT). The feedback is assumed to be causal and noiseless, with a finite capacity constraint $C_{f b}$ in terms of the maximum number of feedback information (bits) per fading block, as illustrated in Fig. 2.

This paper is organized as follows. In Section II, we shall outline the MIMO channel model, the feedback model, and the

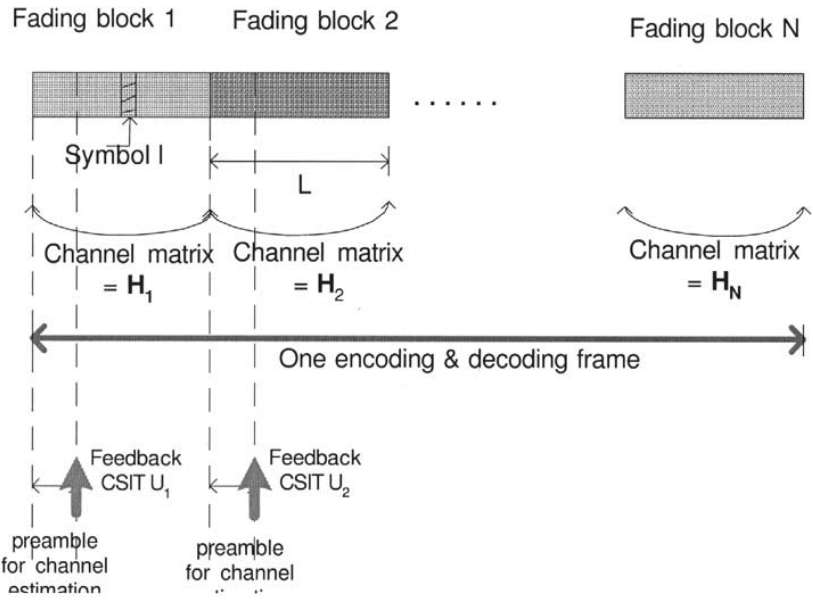

Fig. 2. Encoding and feedback timing block diagram.

hybrid power-control and beamforming model, as well as the problem formulation. In Section III, we shall illustrate that such a hybrid design is indeed the optimal transmission and feedback strategy. In Section IV, we shall illustrate the computationally efficient algorithm for design optimization. In Section V, we present the numerical results and discuss the efficiency of feedback-link capacity for various SNRs and various $n_{T}$ and $n_{R}$. 
Finally, we conclude with a brief summary of results in Section VI.

\section{SYSTEM MODEL}

In this paper, $\mathbf{X}$ denotes a random matrix, while $\mathbf{x}$ denotes a realization of the random matrix. $\mathcal{E}[X]$ denotes unconditional expectation, while $\mathcal{E}_{A}[X]$ denotes conditional expectation (conditioned on the event $A$ ).

\section{A. Forward MIMO Channel Model}

We consider a communication system with a transmitter (with $n_{T}$ transmit antennas) and receiver (with $n_{R}$ receive antennas). Let $\mathbf{X}$ be the $n_{T} \times 1$ transmitted symbol. The $n_{R} \times 1$ received symbol, $\mathbf{Y}$, is given by

$$
\mathbf{Y}=\mathbf{H X}+\mathbf{Z}
$$

where $\mathbf{H}$ is the $n_{R} \times n_{T}$-dimension channel matrix, with element $h_{i, j}$ denoting the zero-mean complex fading between the $i$ th receive antenna and the $j$ th transmit antenna. We assume the antenna separations are sufficiently far apart so that the $\mathcal{E}\left[h_{i, j} h_{l, m}^{*}\right]=0$ for all $(i, j) \neq(l, m)$ and $\mathcal{E}[$.$] denotes ex-$ pectation over all channel realization. Furthermore, we assume $\mathcal{E}\left[\left|h_{i, j}\right|^{2}\right]=1$.

$\mathbf{Z}$ is the $n_{R} \times 1$ channel complex Gaussian noise with covariance matrix $\mathcal{E}\left[\mathbf{Z Z}^{*}\right]=\sigma_{z}^{2} \mathbf{I}_{n_{R}}$. We assume a block-fading channel where the channel-fading matrix remains quasi-static within a fading block, but becomes independent between fading blocks. Channel encoding and decoding frames span a large number of fading blocks. In the limit of a large number of fading blocks, ergodic capacity could be achieved.

\section{B. Feedback Channel Model}

The feedback timing model is illustrated in Fig. 2. For every fading block prior to payload transmission, there is a preamble transmission for CSIR estimation. The channel matrix $\mathbf{H}$ is estimated from the preamble at the receiver. The CSIT symbol, $U \in \mathcal{U}$ (where $\mathcal{U}$ is the set of CSIT symbols), is generated from the estimated channel matrix and fed back to the transmitter. In this paper, we assume the channel matrix is estimated perfectly at the receiver, and this is achievable for asymptotically large fading block sizes [14]. The CSIT is related to the channel matrix in stochastic manner, and is given by the transition probability $p(U \mid \mathbf{H})$, meaning that $U$ is derived stochastically from $\mathbf{H}$. The feedback rate of the CSIT is once per fading block.

We assume the feedback of CSIT is causal in the sense that the transmitter at fading block $n$ has knowledge of the feedback CSIT $\tilde{U}=\left\{U_{1}, \ldots, U_{n}\right\}$ only. Since the channel fading is independent between fading blocks, the transmitted symbol in the current fading block, $\mathbf{X}$, is, therefore, a function of the current feedback CSIT $U$ only. ${ }^{4}$ Due to the causal feedback assumption, only symbol-by-symbol transmission is allowed on the feedback channel. With the causal feedback constraint, no encoding on the CSIT could be done on the feedback channel, because the receiver does not have the future realization of the channel state

\footnotetext{
${ }^{4}$ Since we are dealing with independent and identically distributed (i.i.d.) block-fading channels, knowledge of $\mathbf{h}_{n-1}, \ldots, \mathbf{h}_{0}$ does not provide additional information on $\mathbf{h}_{n}$. Hence, without loss of generality, we assume memoryless feedback.
}

sequence $\tilde{\mathbf{H}}=\left\{\mathbf{H}_{n+1}, \ldots, \mathbf{H}_{\infty}\right\}$ at the $n$th fading block. Furthermore, error-free transmission of feedback information is not possible if the feedback channel is noisy. Since the focus of the paper is to investigate the optimal tradeoff between feedback channel capacity and forward channel capacity, we shall assume the feedback channel is noiseless. The feedback channel is characterized by a capacity constraint, $C_{\mathrm{fb}}$, given by the maximum number of feedback information bits per fading block. We have the following feedback constraint:

$$
\log _{2}|\mathcal{U}| \leq C_{\mathrm{fb}}
$$

where $|\mathcal{U}|$ denotes the cardinality of the CSIT set $\mathcal{U}$ and $C_{\mathrm{fb}}$ is in bits per fading block.

\section{Problem Formulation}

The optimization problem is summarized below.

Problem 1: Given an i.i.d. block-fading MIMO channel with CSIT, $U$, find the optimal transmission strategy (combined beamforming and power control) $\mathcal{E}_{U=u}\left[\mathbf{X X}^{*}\right]$ and feedback strategy $p(U \mid \mathbf{H})$ so that the forward link capacity, $C_{\text {fwd }}$, is maximized and the following constraints are satisfied:

$$
\begin{aligned}
\mathcal{E}\left(\mathbf{X X}^{*}\right) & \leq P_{0} \\
|\mathcal{U}| & \leq 2^{C_{\mathrm{fb}}}
\end{aligned}
$$

where $C_{\mathrm{fb}}$ is the feedback-link capacity and $P_{0}$ is the average transmit power constraint (average over the coding frame).

Please see the next section for the elaboration of the optimization problem strategy.

\section{Hybrid Adaptive Power Control and Beamforming Model}

The proposed feedback strategy and transmission strategy in the presence of feedback-link capacity constraint are illustrated in Fig. 3. At the receiver, the CSIR space is partitioned into $Q$ regions $\left\{\mathcal{H}_{1}, \ldots, \mathcal{H}_{Q}\right\}$ where $Q=2^{C_{\mathrm{fb}}}$. When the current CSIR, $\mathbf{H}$, is in the $q$ th region, $\mathcal{H}_{q}$, the associated partition index, $u_{q}$, is generated and fed back to the transmitter using the feedback channel. (This consumes the feedback-link capacity of $C_{\mathrm{fb}}$.)

At the transmitter, there is a table of $Q$ (power control matrix $\left(\Lambda_{q}\right)$, and beamforming matrix $\left(\mathbf{W}_{q}\right)$ associated with each feedback index $u_{q} \in[1, Q]$. The power control matrix is a diagonal matrix with nonnegative elements. The beamforming matrix is a unitary matrix. When the current CSIT is $u_{q}$, the corresponding power matrix and beamforming matrix are selected for the current fading block. Let $\mathbf{T}$ be the $n_{T} \times 1$ vector of symbol output from the bank of $n_{T}$ encoders. The transmitted symbol $\mathbf{X}$ to the channel at the current fading block, after the application of the power control matrix and the beamforming matrix, is given by

$$
\mathbf{X}=\mathbf{W}_{q} \sqrt{\Lambda_{q}} \mathbf{T} .
$$

Without loss of generality, let $\mathcal{E}\left[\mathbf{T}^{*} \mathbf{T}\right]=1$ for normalization. As will be shown in Section III, the optimal distribution for the channel encoder outputs, $\mathbf{T}$, is complex Gaussian with covariance matrix, $\mathcal{E}\left[\mathbf{T T}^{*}\right]=\mathbf{I}_{n_{T}}$, which is independent of the CSIT.

On the other hand, the corresponding distribution of the channel symbol, $\mathbf{X}$, is complex Gaussian with covariance matrix (depending on CSIT $U_{q}$ ) given by

$$
\mathcal{E}_{U_{q}=u_{q}}\left[\mathbf{X X}^{*}\right]=\mathbf{W}_{q} \Lambda_{q} \mathbf{W}_{q} .
$$




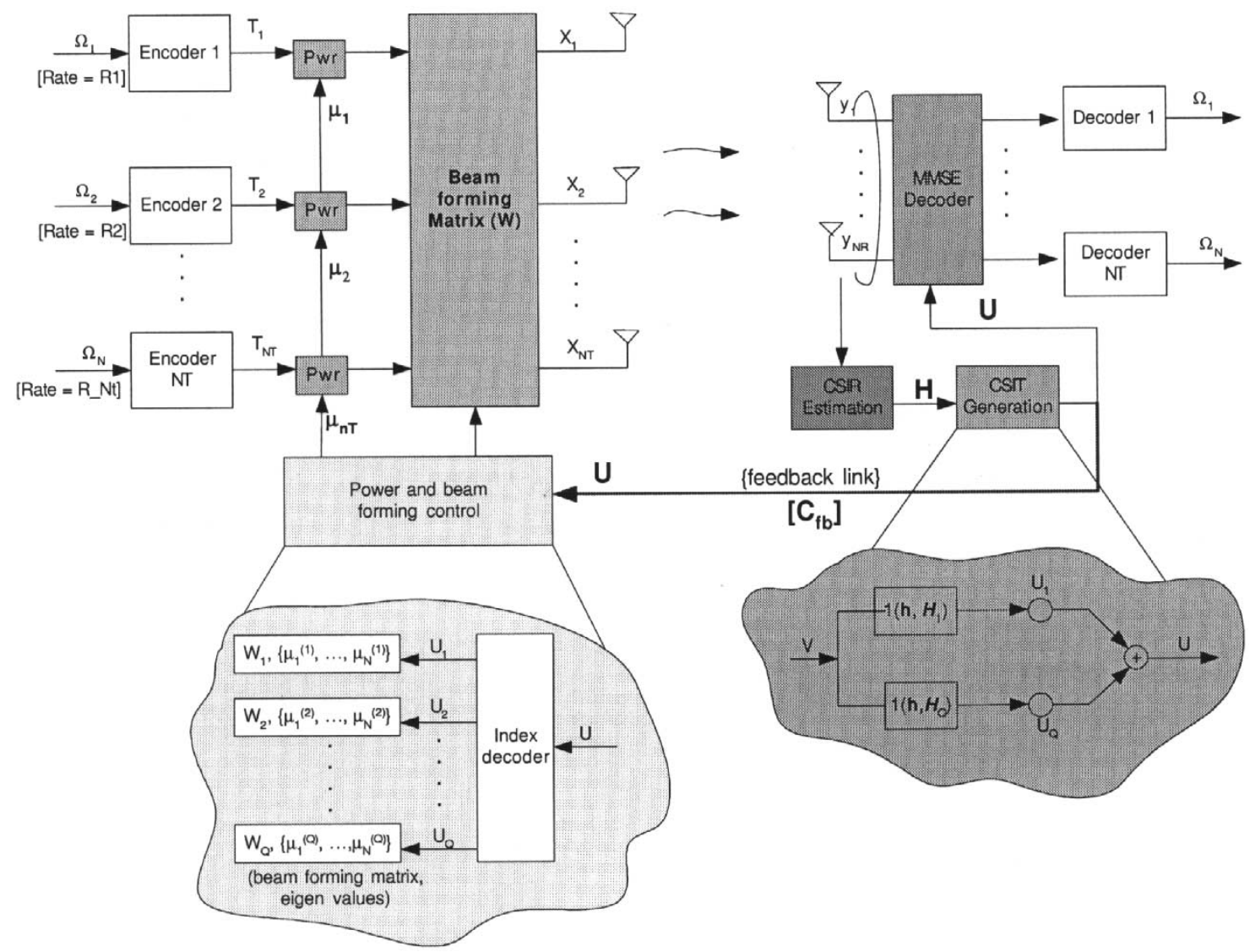

Fig. 3. MIMO transmission strategy with feedback-capacity constraint.

The set of power control matrix and beamforming matrix is chosen such that it satisfies the average transmit power constraint, $P_{0}$. That is

$$
\operatorname{tr} \mathcal{E}\left[\mathbf{W}_{q} \Lambda_{q} \mathbf{W}_{q}\right] \leq P_{0}
$$

where the expectation is over all CSIT realization.

Hence, in the proposed framework, the channel encoding is fixed rate and nonadaptive. Adaptation is applied in the power control matrix and beamforming matrix only. We will show in Section III that this is indeed the optimal feedback and transmission strategies with respect to the problem (1).

\section{Optimal FeEdback Strategy With FEEDBACK-CAPACITY CONSTRAINT}

In this section, we shall show that the proposed structure in Section II-D is the optimal strategy with respect to problem 1. Since the channel noise is complex Gaussian, the capacity achieving distribution of the channel input symbol, $\mathbf{X}$, with CSIT $U$ is complex Gaussian with the conditional covariance matrix satisfying the average transmit power constraint

$$
\operatorname{tr} \mathcal{E}\left(\mathbf{X X}^{*}\right) \leq P_{0}
$$

where the expectation is with respect to $\mathbf{X}$ and CSIT $U$. Given a realization of channel matrix of $\mathbf{H}$ and CSIT of $U$, the conditional channel capacity [15] is given by

$$
C(\mathbf{h})=\max _{p\left(\mathbf{X} \mid U=u_{q}\right)} I(\mathbf{X} ; \mathbf{Y} \mid \mathbf{H}=\mathbf{h})=\log _{2}\left|\mathbf{I}_{n_{T}}+\frac{\mathbf{h}^{*} \mathbf{h} \boldsymbol{\rho}_{q}}{\sigma_{z}^{2}}\right|
$$

Since the covariance matrix of $\mathbf{X}$ is Hermitian, by singular value decomposition, we have

$$
\rho_{q}=\mathcal{E}_{U=u_{q}}\left[\mathbf{X X}^{*}\right]=\mathbf{W}_{q} \Lambda_{q} \mathbf{W}_{q}
$$

where $\Lambda_{q}$ is the diagonal matrix containing eigenvalues of $\rho_{q}$, and $\mathbf{W}_{q}$ is the corresponding eigenmatrix. If we define a $n_{T} \times$ 1-dimensional matrix $\mathbf{T}$ to be complex Gaussian with covariance $\mathbf{I}_{n_{T}}$, we obtain the capacity achieving transmit symbol as

$$
\mathbf{X}=\mathbf{W}_{q} \Lambda_{q} \mathbf{T}
$$

The transmission strategy in (8) is illustrated in the equivalent channel model of Fig. 4. The original adaptive encoding function, which produces complex Gaussian symbols $\mathbf{X}$, from the message index $\omega$, as a function of the CSIT $U$, could be split into a new encoder and a device. The new encoder is independent of CSIT $U$ and maps a message index $\omega$ into a sequence of reference inputs, $\tilde{\mathbf{T}}=\left\{\mathbf{T}_{1}, \ldots, \mathbf{T}_{N}\right\}$, where $\mathbf{T}_{n}$ is the channel input alphabet on the derived channel (dotted line). Since the covariance of $\mathbf{T}$ is $\mathbf{I}_{n_{T}}$, the channel encoder could be represented as a bank of $n_{T}$ scalar encoders, independent of CSIT $U$.

The remaining part of the original encoder becomes an adaptive device, which constitutes the adaptive power control matrix $\left(\Lambda_{q}\right)$ and the adaptive beamforming $\left(\mathbf{W}_{q}\right)$ matrix. Both are functions of CSIT $U$. Hence, comparing the structure in Fig. 3 with Fig. 4, we see that the proposed structure in Section II-D could achieve the optimal capacity.

From Fig. 4, the equivalent channel model becomes a MIMO memoryless channel without feedback, with input $\mathbf{T}$ and out-

where $\rho_{q}=\mathcal{E}_{U=u_{q}}\left[\mathbf{X X}^{*}\right]$ is the conditional covariance matrix. 


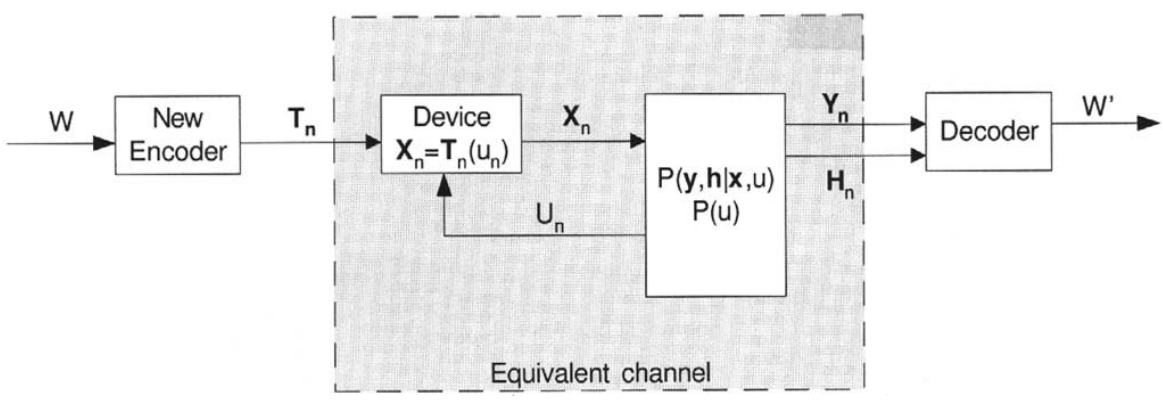

Fig. 4. Equivalent channel model of the communication system with CSIT and CSIR.

puts $(\mathbf{Y}, \mathbf{H})$. Hence, the forward ergodic channel capacity is, therefore, given by

$$
C_{\mathrm{fwd}}=\max _{\left\{\rho_{1}, \ldots, \rho_{Q}\right\}} I(\mathbf{T} ; \mathbf{Y}, \mathbf{H}) .
$$

We shall next consider the set of all feasible feedback strategies satisfying the feedback-link constraint. This is explained in the following lemma.

Lemma 1: The set of feedback strategies $\{p(u \mid \mathbf{h})\}$ that optimizes the forward channel capacity must be deterministic. That is, $u=g(\mathbf{h})$ for some deterministic function $g: \mathcal{H} \rightarrow \mathcal{U}$.

Proof 1: Intuitively, a stochastic relationship between $u$ and $\mathbf{h}$ is equivalent to feedback error. Hence, the set of optimal feedback strategies must be deterministic. The formal proof is illustrated in Appendix A.

From the results of Lemma 1 the CSIT is a deterministic function of the channel state. Hence, the forward capacity in (9) could be simplified [15] to

$$
C_{\mathrm{fwd}}=\mathcal{E}_{\mathbf{h}, u}\left[\max _{\{\rho(u)\}} I(\mathbf{X} ; \mathbf{Y} \mid \mathbf{H}=\mathbf{h})\right] .
$$

The joint distribution of the optimizing feedback strategy, $p(u, \mathbf{h})$, is given by

$$
p(u, \mathbf{h})=\sum_{q=1}^{Q} \delta\left(u-u_{q}\right) p(\mathbf{h}) \mathbf{1}\left(\mathbf{h} \in \mathcal{H}_{q}\right)
$$

where $\mathbf{1}(w)=1$ if $w$ is true, and $\mathbf{1}(w)=0$ if $w$ is false and $\left\{\mathcal{H}_{1}, \ldots, \mathcal{H}_{Q}\right\}$ is the partition on the channel matrix space $\mathcal{H}$.

Hence, the optimal transmission and feedback strategy is given by the proposed structure in Section II-D. The feedback strategy is completely characterized by the partition set $\tilde{\mathcal{H}}=\left\{\mathcal{H}_{1}, \ldots, \mathcal{H}_{Q}\right\}$. The transmission strategy is characterized by the covariance matrices $\left\{\rho_{1}, \ldots, \rho_{Q}\right\}$. The optimization problem in (1) for the forward channel capacity is equivalent to the following.

Problem 2: Find a feedback strategy $\tilde{\mathcal{H}}=\left\{\mathcal{H}_{1}, \ldots, \mathcal{H}_{Q}\right\}$, as well as a transmission strategy $\left\{\rho_{1}, \ldots, \rho_{Q}\right\}$, such that $\operatorname{tr} \mathcal{E}\left[\rho_{q}\right] \leq P_{0}$ and the ergodic forward capacity is maximized

$$
C_{\mathrm{fwd}}=\sum_{q=1}^{Q} \max _{\left\{\rho_{q}, \mathcal{H}_{q}\right\}} \mathcal{E}_{\mathbf{h} \in \mathcal{H}_{q}}\left[\log _{2}\left|\mathbf{I}_{n_{T}}+\frac{\mathbf{h}^{*} \mathbf{h} \rho_{q}}{\sigma_{z}^{2}}\right|\right] P_{q}
$$

where $\mathcal{E}_{\mathbf{h} \in \mathcal{H}_{q}}[\mathbf{x}]$ denotes the conditional expectation (conditional on $\mathbf{h} \in \mathcal{H}_{q}$ ) and $P_{q}=\operatorname{Pr}\left[\mathbf{h} \in \mathcal{H}_{q}\right]$.

\section{THE OPTIMAL SOLUTION}

We shall illustrate that the design of the optimal feedback strategy $\tilde{\mathcal{H}}=\left\{\mathcal{H}_{1}, \ldots, \mathcal{H}_{Q}\right\}$, and transmission strategy $\left\{\rho_{1}, \ldots, \rho_{Q}\right\}$, is equivalent to the design of a vector quantizer with a modified distortion measure.

Including the Lagrange multiplier $\lambda$ for the average transmit power constraint, define a distortion measure for optimizing the forward channel capacity as $d_{\text {cap }}\left(\mathbf{h}, \rho_{q}\right)$. It is given by

$$
d_{\text {cap }}\left(\mathbf{h}, \rho_{q}\right)=-\left[\log _{2}\left|\mathbf{I}_{n_{T}}+\frac{\mathbf{h}^{*} \mathbf{h} \rho_{\mathrm{q}}}{\sigma_{z}^{2}}\right|-\lambda \operatorname{tr} \rho_{\mathrm{q}}\right] .
$$

Observe that the distortion measure is a function of $\mathbf{h}$ and $\rho_{q}$ only. The partition index $u_{q}$ becomes a dummy variable and is omitted in the formulation.

The optimization problem in (11) is equivalent to selecting $\left\{\rho_{1}, \ldots, \rho_{Q}\right\}$ and $\tilde{\mathcal{H}}=\left\{\mathcal{H}_{1}, \ldots, \mathcal{H}_{Q}\right\}$ so as to minimize the average distortion $D_{\text {cap }}$ given by

$$
D_{\text {cap }}=\sum_{q=1}^{Q} \mathcal{E}_{\mathbf{h} \in \mathcal{H}_{q}}\left[d_{\text {cap }}\left(\mathbf{h}, \rho_{q}\right)\right] P_{q} .
$$

Hence, the optimization problems could be solved by Lloyd's algorithm, which is outlined below. Step 1 and Step 2 are repeatedly applied until convergence. ${ }^{5}$ Note that the algorithm below is an offline optimization algorithm, and hence, complexity has a relatively small impact on implementation. The online strategy is given by Section II-D and has a very low runtime complexity.

Step 1) Determine the optimal transmission strategy $\left.\left\{\rho_{1}, \ldots, \rho_{Q}\right)\right\}$ given a certain partition, $\left\{\mathcal{H}_{1}, \ldots, \mathcal{H}_{Q}\right\}$.

The optimal transmission strategy, $\rho_{q}$, is given by the generalized partition centroid

$$
\rho_{q}=\arg \min _{\rho_{q}} \mathcal{E}_{\mathbf{h} \in \mathcal{H}_{q}}\left[d\left(\mathbf{h}, \rho_{q}\right)\right] P_{q} .
$$

Step 2) Determine the optimal partition $\left\{\mathcal{H}_{q}\right\}$ given a transmission strategy $\left\{\rho_{q}\right\}$.

The optimal partition is given by the nearest neighbor rule

$$
\mathcal{H}_{q}=\left\{\mathbf{h}: d\left(\mathbf{h}, \rho_{q}\right) \leq d\left(\mathbf{h}, \rho_{j}\right) ; \forall j, q \in[1, \ldots, Q], j \neq q\right\} .
$$

5In general, there is no guarantee that Lloyd's algorithm will converge to the global optimal [16]. In the simulation, we repeat each partition iteration 10 times, each time starting with a random set of initial partition centroids. At the end of the algorithm, we pick the one that gives us the largest capacity. 


\section{A. Approximate Closed-Form Solution for Step 1}

To solve for (14), we let $f=\mathcal{E}_{\mathbf{h} \in \mathcal{H}_{q}}\left[d_{\text {cap }}\left(\mathbf{h}, \rho_{q}\right)\right] P_{q}$. As a result of the expectation operator, the optimization of the above equation is quite tedious. In order to have closed-form solutions for Step 1, we shall consider the following heuristic approximation:

$$
\begin{aligned}
f & =\mathcal{E}_{\mathbf{h} \in \mathcal{H}_{q}}\left[\log _{2}\left|\mathbf{I}_{n_{T}} \frac{\mathbf{h}^{*} \mathbf{h} \rho_{q}}{\sigma_{z}^{2}}\right|-\lambda \operatorname{tr}\left(\rho_{q}\right)\right] P_{q} \\
& \approx P_{q}\left[\log _{2}\left|\mathbf{I}_{n_{T}}+\frac{\overline{\mathbf{s}} \rho_{q}}{\sigma_{z}^{2}}\right|-\lambda \operatorname{tr}\left(\rho_{q}\right)\right]
\end{aligned}
$$

where $\overline{\mathbf{s}}=\mathcal{E}_{\mathbf{h} \in \mathcal{H}_{q}}\left[\mathbf{h}^{*} \mathbf{h}\right]$ is a conditional average matrix and $P_{q}=\operatorname{Pr}\left[\mathbf{h} \in \mathcal{H}_{q}\right]$ for the $q$ th partition.

The approximate closed-form solution derived below as a result of the approximation in (16) serves as a realizable, nontrivial capacity lower bound.

Since $\overline{\mathbf{s}}$ is Hermitian, it could be uniquely expressed as $\overline{\mathbf{s}}=$ $\overline{\mathbf{w}} \bar{\Psi}_{s} \overline{\mathbf{w}}^{*}$, where $\bar{\Psi}_{v}$ is the diagonal matrix of eigenvalues of $\overline{\mathbf{s}}$, and $\overline{\mathbf{w}}$ is the unitary matrix of the corresponding eigenvectors. Multiplying $\overline{\mathbf{w}}^{*}$ (to the left) and $\overline{\mathbf{w}}$ (to the right) of the determinant on the right-hand side of (16), we have

$$
\left|\mathbf{I}_{n_{T}}+\frac{\overline{\mathbf{s}} \rho_{q}}{\sigma_{z}^{2}}\right|=\left|\overline{\mathbf{w}}^{*} \overline{\mathbf{w}}+\frac{\overline{\mathbf{w}}^{*} \overline{\mathbf{s}} \rho_{q} \overline{\mathbf{w}}}{\sigma_{z}^{2}}\right|=\left|\mathbf{I}_{n_{T}}+\frac{\bar{\Psi}_{s} \overline{\mathbf{w}}^{*} \rho_{q} \overline{\mathbf{w}}}{\sigma_{z}^{2}}\right| \text {. }
$$

Observe that in general, the optimizing $\rho_{q}$ has the form

$$
\rho_{q}=\overline{\mathbf{w}} \Psi_{\rho} \overline{\mathbf{w}}^{*}
$$

because this would diagonalize the argument of the determinant, and therefore, maximize the determinant.

Hence, we have $\operatorname{tr}\left(\rho_{q}\right)=\operatorname{tr}\left(\Psi_{\rho}\right)$. Let $\mathcal{A}$ be the set of indexes with nonzero entries in $\bar{\Psi}_{s}$. That is

$$
\mathcal{A}=\left\{t: \bar{\Psi}_{s}(t, t)>0\right\} \text {. }
$$

Equation (16) could be simplified as

$$
f=\sum_{t \in \mathcal{A}} \log _{2}\left(1+\frac{\bar{\Psi}_{s}(t, t) \Psi_{\rho}(t, t)}{\sigma_{z}^{2}}\right)-\lambda \operatorname{tr}\left(\Psi_{\rho}\right) .
$$

By observation, $\Psi_{\rho}(t, t)=0$ if $t \notin \mathcal{A}$. For $t \in \mathcal{A}$, the necessary condition for optimal point is given by $d f / d \Psi_{\rho}(t, t)=0$. This is equivalent to

$$
\left(1+\frac{\bar{\Psi}_{s}(t, t) \Psi_{\rho}(t, t)}{\sigma_{z}^{2}}\right)^{-1} \bar{\Psi}_{s}(t, t)-\lambda=0
$$

for all $t \in \mathcal{A}$. The optimal solution is given by

$$
\Psi_{\rho}=\left[\frac{\mathbf{I}}{\lambda}-\sigma_{z}^{2} \bar{\Psi}_{s}^{-1}\right]^{+}
$$

where $[x]^{+}=\max (x, 0)$.

The corresponding optimal input covariance matrix $\rho_{q}$ is given by $\rho_{q}=\overline{\mathbf{w}} \Psi_{\rho} \overline{\mathbf{w}}^{*}$.

The average transmitted power over the coding block is given by

$$
\bar{\rho}=\sum_{q=1}^{Q} P_{q} \operatorname{tr}\left[\rho_{q}\right] .
$$

The corresponding average SNR is given by $\left(\bar{\rho} / \sigma_{z}^{2}\right)$. Note that from (21), the optimal feedback strategy with partial feedback constraint also has the form of temporal waterfilling.

\section{B. MMSE-SIC Receiver Structure}

At the receiver, MMSE-SIC could be used to achieve the optimal channel capacity. From (1), the received symbol $\mathbf{Y}$ is given by

$$
\begin{aligned}
\mathbf{Y} & =\mathbf{H X}+\mathbf{Z}=\mathbf{H} \mathbf{W} \sqrt{\Psi_{\rho}} \mathbf{T}+\mathbf{Z} \\
& =\sum_{m=1}^{n_{T}} \mathbf{F}_{m} \sqrt{\mu_{m}} T_{m}+\mathbf{Z}
\end{aligned}
$$

where $\mathbf{F}=\mathbf{H W}$ represents the aggregate effect of matrix channel and beamforming matrix, $\mathbf{T}$ is the $n_{T} \times 1$-dimension i.i.d. complex Gaussian input vector from the channel encoder bank, $T_{m}$ is the $m$ th component of $\mathbf{T}$, and $\mu_{m}=\Psi_{\rho}(m, m)$ is the $m$ th diagonal element representing the power control action.

In fact, the MMSE spatial processing [17] stems from the chain rule of mutual information, which is illustrated below. From the structure in Fig. 3, the channel capacity is given by

$$
\begin{aligned}
C_{\mathrm{fwd}} & =\sum_{q=1}^{Q} \mathcal{E}_{\mathbf{h} \in \mathcal{H}_{q}}[I(\mathbf{T} ; \mathbf{Y} \mid \mathbf{H})] \\
& =\sum_{m=1}^{n_{T}} \sum_{q=1}^{Q} \mathcal{E}_{\mathbf{h} \in \mathcal{H}_{q}}\left[I\left(T_{m} ; \mathbf{Y} \mid T_{1}, \ldots, T_{m-1}, \mathbf{H}\right)\right] \\
& =\sum_{q=1}^{Q} \sum_{m=1}^{n_{T}} R_{m}
\end{aligned}
$$

where $R_{m}$ is the encoding rate of the $m$ th encoding branch at the transmitter, given by

$$
\begin{aligned}
R_{m}=\sum_{q=1}^{Q} \mathcal{E}_{\mathbf{h} \in \mathcal{H}_{q}}\left[I\left(T_{m} ; \mathbf{Y} \mid T_{1}, \ldots, T_{m-1}, \mathbf{H}\right)\right] \\
=\sum_{q=1}^{Q} \mathcal{E}_{\mathbf{h} \in \mathcal{H}_{q}}\left[\operatorname { l o g } _ { 2 } \left(1+\frac{\mu_{m}}{\sigma_{z}^{2}} \mathbf{F}_{m}^{*}\left(\mathbf{I}_{n_{R}}\right.\right.\right. \\
\left.\left.\left.\quad+\sum_{i=m+1}^{n_{T}} \frac{\mu_{i}}{\sigma_{z}^{2}} \mathbf{F}_{i} \mathbf{F}_{i}^{*}\right)^{-1} \mathbf{F}_{m}\right)\right] .
\end{aligned}
$$

Hence, the MMSE-SIC processing at the receiver is based on SIC. The information from encoder 1 (with rate $R_{1}$ ) is first decoded, and then the signal is subtracted from the received symbol $\mathbf{Y}$. The information from encoder 2 (with rate $R_{2}$ ) is then decoded without the interference from encoder 1 . The process continues until all the information from the $n_{T}$ channel encoders are decoded. Note that the decoding order is irrelevant in the formulation, but has to be agreed between the transmitter and the receiver. Furthermore, due to coding frame spans over multiple fading blocks, the rate information $\left\{R_{1}, \ldots, R_{n_{T}}\right\}$ is deterministic and could be precomputed at the transmitter without extra feedback.

\section{Feedback Performance}

In this section, we shall illustrate that the SNR gain of the partial feedback is contributed to by two factors, namely, the temporal waterfilling and the spatial waterfilling. In general, the SNR gain of perfect CSIT relative to no CSIT is shown to be lower bounded by $10 \log _{10}\left(n_{T} / n_{R}\right) \mathrm{dB}$. 


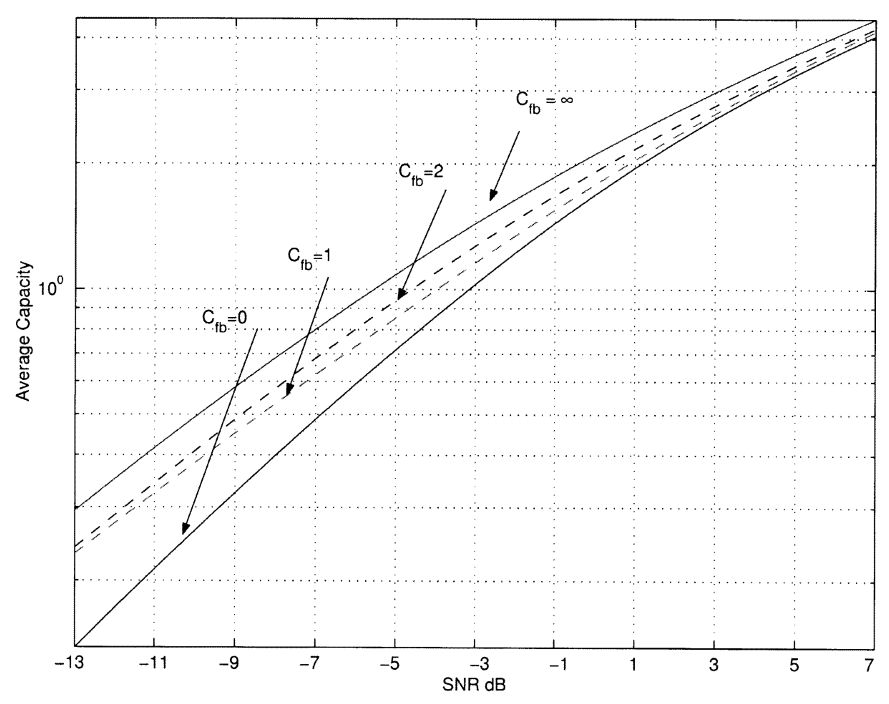

Fig. 5. Optimal channel capacity versus average SNR of $2 \times 2$ system at various feedback channel capacities $C_{f b}=0,1,2, \infty$.

Lemma 2: If $n_{T}>n_{R}$, the SNR gain of full feedback versus no feedback for the MIMO system is bounded below from $10 \log _{10}\left(n_{T} / n_{R}\right)$.

Proof 2: Please refer to [18].

\section{RESULTS AND DISCUSSIONS}

We shall evaluate the performance of the partial feedback design in Section IV-A with numerical results in this section. We shall consider the SNR gain of the forward channel capacity with various $n_{T}$ and $n_{R}$ configurations and various SNRs. When feedback capacity $C_{\mathrm{fb}}=0$, it corresponds to the case with no CSIT. On the other hand, when the feedback capacity $C_{\mathrm{fb}}=\infty$, it corresponds to the case with perfect CSIT. Note that $C_{\mathrm{fb}}$ has a unit of bit per fading block.

\section{A. Performance of MIMO Link}

We shall illustrate the contribution of temporal power waterfilling ${ }^{6}$ to the overall SNR gain in this section. A $2 \times 2$ MIMO link performance is studied. Fig. 5 illustrates the $2 \times 2$ forward MIMO channel capacity versus the average forward SNR, with feedback capacity constraint varying from $C_{\mathrm{fb}}=0$ to $C_{\mathrm{fb}}=\infty$. For low SNRs, there is a significant SNR gain of around 3.5 $\mathrm{dB}$ in forward channel capacity between perfect CSIT and no CSIT. Furthermore, there is a gain of 2.5 and $3 \mathrm{~dB}$ for partial feedback with feedback capacity $C_{\mathrm{fb}}=1$ and $C_{\mathrm{fb}}=2$, respectively. In other words, $C_{\mathrm{fb}}=2$ realized about $85 \%$ of the feedback gain. On the other hand, the effectiveness of feedback is reduced (but still significant) for high SNRs. This is reasonable, because when the average SNR is large, the penalty of transmitting power less efficiently is small compared with the case when the average SNR is small.

\footnotetext{
${ }^{6}$ Although the transmit power and beamforming matrix is constant within a fading block, they are adaptive to the partial feedback once every fading block, and therefore, we have temporal adaptation over a time horizon of a coding frame.
}

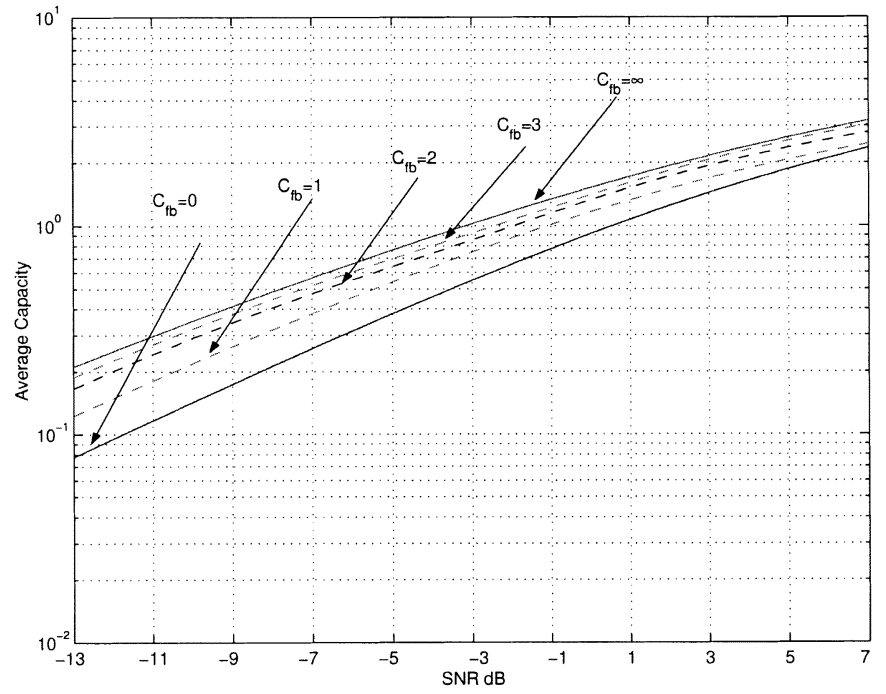

(a)

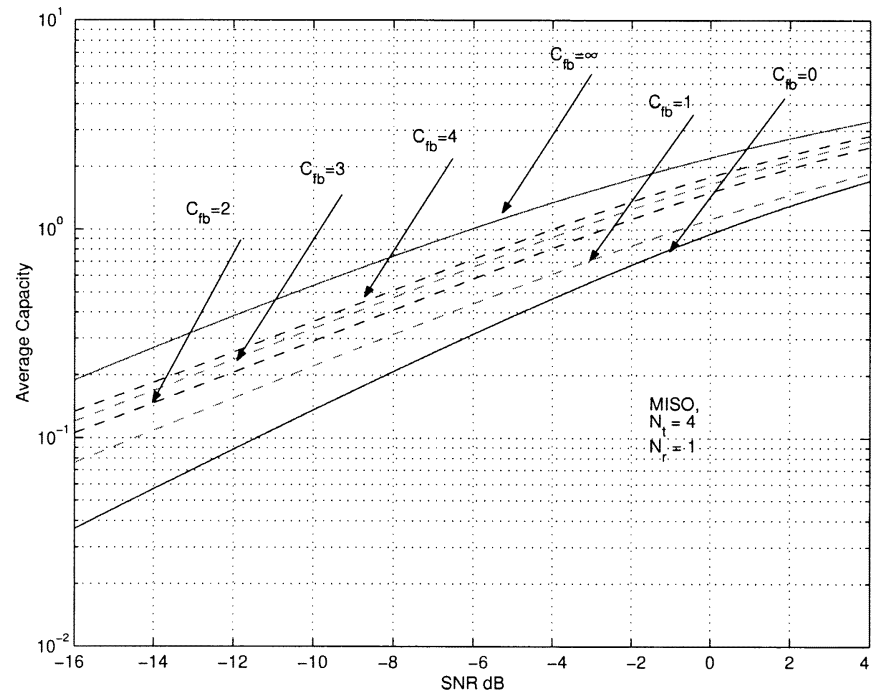

(b)

Fig. 6. Forward channel capacity versus average SNR with ideal CSIR for 2 $\times 1$ and $4 \times 1$ systems and $C_{\mathrm{fb}}=0,1,2,3,4, \infty$.

\section{B. Performance of MISO Link}

In this section, we consider $n_{T}=2,4$ and $n_{R}=1$. Fig. 6(a) and (b) illustrate the forward channel capacity versus average SNR with $C_{\mathrm{fb}}=0$ to $C_{\mathrm{fb}}=\infty,\left(n_{T}, n_{R}\right)=(2,1)$, and $\left(n_{T}, n_{R}\right)=(4,1)$, respectively. For low SNRs, there are significant SNR gains of around 5 and $8 \mathrm{~dB}$ in forward channel capacity between perfect CSIT and no CSIT for $n_{T}=2$ and $n_{T}=$ 4 , respectively. For $n_{T}=2$, there is a gain of $2.2,4$, and $4.4 \mathrm{~dB}$ for partial feedback with feedback capacity $C_{\mathrm{fb}}=1,2,3$, respectively. For $n_{T}=4$, the corresponding SNR gains are 2.4, 4,5 , and $5.5 \mathrm{~dB}$ for feedback capacity of $C_{\mathrm{fb}}=1,2,3,4$, respectively.

For high SNRs, there are also significant SNR gains of 3 and $6 \mathrm{~dB}$ between the full feedback and no feedback case for $n_{T}=2$ and $n_{T}=4$, respectively. These results demonstrate that the spatial power waterfilling is very effective for high and low SNRs for $2 \times 1$ and $4 \times 1$ systems. There is a large incremental SNR gain from $C_{\mathrm{fb}}=1$ to $C_{\mathrm{fb}}=2$. 


\section{CONCLUSION}

In this paper, we proposed a combined adaptive power control and beamforming transmission strategy for MIMO i.i.d. block-fading channels with feedback. The feedback channel is causal and has a feedback capacity constraint $\left(C_{\mathrm{fb}}\right)$ in terms of the maximum number of feedback bits per fading block. Channel matrix is estimated at the receiver, and a CSIT $U$ is fed back to the transmitter. We show that the proposed scheme could achieve the optimal partial feedback capacity, and the design of the optimal feedback scheme is identical to the design of vector quantizer (Lloyd's algorithm [16]) with a modified distortion measure. In general, the performance of feedback is contributed by temporal and spatial power waterfilling. The former factor enhances the forward channel capacity more effectively for low SNR, while the latter factor enhances the forward channel capacity effectively for both high and low SNR values, especially when $n_{T} \gg n_{R}$.

\section{APPENDIX A \\ PROOF OF LEMMA 1}

Observe that $I(\mathbf{T}, U ; \mathbf{Y} \mid \mathbf{H})=I(\mathbf{T} ; \mathbf{Y} \mid U, \mathbf{H})+I(U ; \mathbf{Y} \mid \mathbf{H})$. Similarly, $I(\mathbf{T}, U ; \mathbf{Y} \mid \mathbf{H})=I(\mathbf{T} ; \mathbf{Y} \mid \mathbf{H})+I(U ; \mathbf{Y} \mid \mathbf{T}, \mathbf{H})$. Hence, we have

$$
\begin{aligned}
I(\mathbf{T} ; \mathbf{Y} \mid \mathbf{H})= & I(\mathbf{T} ; \mathbf{Y} \mid U, \mathbf{H})+I(U ; \mathbf{Y} \mid \mathbf{H}) \\
& -I(U ; \mathbf{Y} \mid \mathbf{T}, \mathbf{H}) \\
= & I(\mathbf{T} ; \mathbf{Y} \mid U, \mathbf{H})+I(U ; \mathbf{T} \mid \mathbf{H}) \\
& -I(U ; \mathbf{T} \mid \mathbf{Y}, \mathbf{H}) .
\end{aligned}
$$

Note that $I(U ; \mathbf{T} \mid \mathbf{H})=0$, because $U$ and $\mathbf{T}$ are conditionally independent given $\mathbf{H}$. Hence, we have $I(\mathbf{T} ; \mathbf{Y} \mid \mathbf{H})=$ $I(\mathbf{T} ; \mathbf{Y} \mid U, \mathbf{H})-I(U ; \mathbf{T} \mid \mathbf{H}, \mathbf{Y})$.

If $u=g(\mathbf{h})$ is a deterministic function, we have $I(U ; \mathbf{T} \mid \mathbf{Y}, \mathbf{H})=0$. Therefore, we have

$$
I_{\mathrm{det}}=I(\mathbf{T} ; \mathbf{Y} \mid \mathbf{H})=I(\mathbf{T} ; \mathbf{Y} \mid U, \mathbf{H}) .
$$

On the other hand, if $u=g(\mathbf{h})$ is a random function, we have

$$
I_{\mathrm{ran}}=I(\mathbf{T} ; \mathbf{Y} \mid \mathbf{H}) \leq I(\mathbf{T} ; \mathbf{Y} \mid U, \mathbf{H})=I_{\mathrm{det}}
$$

with equality held when $u=g(\mathbf{h})$ is deterministic. As a result of the above and (9), the optimal feedback strategy must be given by a deterministic function, $u=g(\mathbf{h})$.

\section{REFERENCES}

[1] L. H. Ozarow, S. Shamai, and A. D. Wyner, "Information theoretic considerations for cellular mobile radio," IEEE Trans. Veh. Technol., vol. 43, pp. 359-378, May 1994.

[2] C. E. Shannon, "Channels with side information at the transmitter," IBM J. Res. Develop., pp. 289-293, 1958.

[3] T. M. Cover and J. A. Thomas, Elements of Information Theory, 2nd ed. New York: Wiley, 1991
[4] K. N. Lau, "Variable rate adaptive channel coding for wireless commmunications," Ph.D. dissertation, Cambridge Univ., Cambridge, U. K., Sept. 1997.

[5] A. J. Goldsmith and P. Varaiya, "Capacity of fading channels with channel side information," IEEE Trans. Inform. Theory, vol. 43, pp. 1986-1992, Nov. 1997.

[6] K. N. Lau, "Variable rate adaptive channel coding for DS-CDMA," Bell Labs Tech. J., pp. 311-316, Nov. 2000.

[7] K. J. Hole and G. E. Oien, "Spectral efficiency of adaptive coded modulation in urban microcellular networks," IEEE Trans. Veh. Technol., vol. 50, pp. 205-222, Jan. 2001.

[8] E. Telatar, "Capacity of multiple antenna Gaussian channels," Eur. Trans. Commun., vol. 44, pp. 2619-2692, Oct. 1999.

[9] A. Moustakas and S. H. Simon, Optimizing multi-transmitter singlereceiver (MISO) antenna systems with partial channel knowledge, in Lucent Internal Tech. Memo, 2002.

[10] K. N. Lau, Y. J. Liu, and T. A. Chen, "On the role of transmit diversity in wireless communications with partial feedback," IEEE Trans. Commun., vol. 50, pp. 2082-2090, Dec. 2002.

[11] J. C. Roh and B. D. Rao, "An improved transmission strategy for multiple antenna channels with partial feedback," in Proc. Asilomar Conf. Signals, Systems, Computers, Nov. 2002, pp. 609-613.

[12] S. Zhou and G. B. Giannakis, "Optimal transmitter eigen-beamforming and space-time block code based on channel mean feedback," IEEE Trans. Signal Processing, vol. 50, pp. 2599-2613, Oct. 2002.

[13] 3GPP Tech. Spec. G. T. 25-848, Mar. 2001

[14] B. Hassibi and B. M. Hochwald, "How much training is needed in multiple-antenna wireless links," IEEE Trans. Inform. Theory, submitted for publication.

[15] G. Caire and S. Shamai, "On the capacity of some channels with channel state information," IEEE Trans. Inform. Theory, vol. 45, pp. 2007-2019, Sept. 1999.

[16] S. P. Lloyd, "Least-square quantization in PCM," IEEE Trans. Inform. Theory, vol. IT-28, pp. 129-137, Mar. 1982.

[17] S. Verdu, Multiuser Detection. Cambridge, U. K.: Cambridge Univ. Press, 1998.

[18] K. N. Lau, Optimal partial feedback design for block fading channels-Part II: MIMO channels, in Bell Labs Tech. Memo., Aug. 2002.

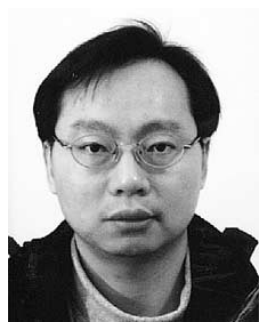

Vincent Lau (M'97-SM'01) was born in Hong Kong. He received the undergraduate degree in electrical and electronics engineering with distinction (first-class honors) from the University of Hong Kong, Hong Kong, in 1992, and the Ph.D. degree from Cambridge University, Cambridge, U.K. in 1997.

He joined Lucent Technologies, Whippany, NJ, as a Member of Technical Staff in 1997. He left Lucent and joined the University of Hong Kong in 1999 as an Assistant Professor, and was later appointed the Codirector of Information Engineering Programmes as well as the Codirector of the 3G Wireless Technology Center. He left the University and rejoined Lucent in July 2001. In 2003, he joined the Department of Electrical and Electronic Engineering, University of Hong Kong. He was appointed the Co-Director of the Information Engineering program, as well as the Co-Director of the $3 \mathrm{G}$ Wireless Technology Center. At the same time, he is a senior manager of HK-ASTRI, leading a team of architecture and algorithm for wireless LAN access infrastructure with smart antenna. His research interests include digital transceiver design, adaptive modulation and channel coding, CDMA power control, soft handoff and CREST factor control algorithms, jointly adaptive multiple access protocols, and short-range wireless ad hoc networking. He has published more than 80 papers in IEEE conference proceedings and journals, and he is the principal author of a book on MIMO technologies, as well as the author of a book chapter on wideband CDMA technologies. In addition, he has eight U.S. patents pending.

Dr. Lau was the invited Session Chair of the IEEE WCNC 2000 International Conference, the IEEE CAS 3G Workshop 2000, and the SCT 2001 International Conference. He received two Best Paper Awards from the Institution of Electrical Engineers and the Hong Kong Institute of Engineers (HKIE) in 1999. He was awarded the Sir Edward Youde Memorial Fellowship and an award from the Croucher Foundation in 1995. 
Youjian (Eugene) Liu (S'98-M'01) was born in Kunming, Yunnan, China. He received the M.S. and Ph.D. degrees in electrical engineering from Ohio State University, Columbus, in 1998 and 2001, respectively, the M.S. degree in electronics from Beijing University, Beijing, China, in 1996, and the B.E. degree in electrical engineering from Beijing University of Aeronautics and Astronautics, Beijing, China, in 1993.

Since August 2002, he has been an Assistant Professor in the Department of Electrical and Computer Engineering, University of Colorado at Boulder. From January 2001 to August 2002, he was a member of the Technical Staff in the CDMA System Analysis and Algorithms Group, Wireless Advanced Technology Laboratory, Lucent Technologies, Bell Labs Innovations, Whippany, NJ. His research interests include communication, coding theory, and information theory.

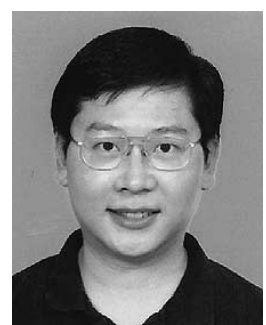

Tai-Ann Chen (S'96-M'98) received the B.S.E.E. and B.S.I.E. double-major degrees with the highest honor from National Tsing Hua University, Hsinchu, Taiwan, in 1989, and the M.S.E.E. and Ph.D. degrees from the School of Electrical and Computer Engineering, Purdue University, West Lafayette, IN, in 1994 and 1998, respectively.

He has been with the Wireless Advanced Technology Labs, Lucent Technologies, Whippany, NJ, since 1998 as a Member of Technical Staff. He is currently working on the design and analysis of 3G UMTS/CDMA2000 systems. His research interests include space-time fading channel characteristics, multiple-antenna techniques, physical layer communication theory, and information theory. In addition to various paper publications, he has also contributed to three U.S. patents.

Dr. Chen was the recipient of the IEEE Leonard G. Abraham Prize Paper Award in 2001 as being the primary author of the selected best paper in the IEEE JOURNAL OF SELECTED AREAS IN COMMUNICATIONS. He is a member of the IEEE Communication Society and the IEEE Information Theory Society, and is a Planning Committee Member of the Wireless and Optical Conference (WOCC). 